\title{
TANGENTIAL DEFORMATIONS ON FIBRED POISSON MANIFOLDS*
}

\author{
D. ARNAL ${ }^{\dagger}$, N. DAHMENE ${ }^{\ddagger}$, A. GAMMELLA $^{\S}$, AND M. MASMOUDI ${ }^{\uparrow}$
}

\begin{abstract}
In a recent article, Cattaneo, Felder and Tomassini explained how the notion of formality can be used to construct flat Fedosov connections on formal vector bundles on a Poisson manifold $M$ and thus a star product on $M$ through the original Fedosov method for symplectic manifolds. In this paper, we suppose that $M$ is a fibre bundle manifold equipped with a Poisson tensor tangential to the fibers. We show that in this case the construction of Cattaneo-FelderTomassini gives tangential (to the fibers) star products.
\end{abstract}

Key words. Deformation quantization, tangentiality, Poisson manifold

AMS subject classifications. 53D 55, 53D17, 53C12, 58F 18

1. Introduction. In order to prove the existence of a deformation quantization (star product) for any Poisson manifold, M. Kontsevich introduced the notion of formality $\mathcal{F}$ and etablished in $[\mathrm{K}]$ that on each manifold there exists formalities, starting from a totally explicit formality $\mathcal{U}$ for $\mathbb{R}^{d}$.

If $\mathcal{F}=\sum \mathcal{F}_{k}$ is a formality on a manifold $M$, then for any Poisson structure $\alpha$ of $M$, the expression

$$
\star=\sum_{k \geq 0} \frac{\hbar^{k}}{k !} \mathcal{F}_{k}(\alpha \ldots \ldots \alpha)
$$

defines a star product on $(M, \alpha)$. In fact, the formality gives much more objects. For instance, it is possible to consider the formal series

$$
\begin{aligned}
A(\xi, \alpha) & =\sum_{k \geq 0} \frac{\hbar^{k}}{k !} \mathcal{F}_{k+1}(\xi \bullet \alpha \ldots \ldots \alpha) \\
F(\xi, \eta, \alpha) & =\sum_{k \geq 0} \frac{\hbar^{k}}{k !} \mathcal{F}_{k+2}(\xi \bullet \eta \ldots \ldots \alpha) \\
R(f, \alpha) & =\sum_{k \geq 0} \frac{\hbar^{k}}{k !} \mathcal{F}_{k+1}(f \cdot \alpha \ldots \ldots \alpha)
\end{aligned}
$$

where $\xi, \eta$ are vector fields and $f$ is a smooth function on $M$, and the formality equation gives many relations between these objects.

Thus, starting only with the 'canonical' formality for $\mathbb{R}^{d}$, Cattaneo, Felder and Tomassini obtain in [CFT] an explicit construction of a star product on an arbitrary Poisson manifold $(M, \alpha)$. More precisely, they first build on $M$ a formal vector bundle

\footnotetext{
*Received March 29, 2002; accepted for publication November 16, 2004.

†Université de Bourgogne, Institut de Mathématiques de Bourgogne, BP 47870, 21078 Dijon Cedex, France (didier.arnal@u-bourgogne.fr).

${ }^{\ddagger}$ Faculté des sciences de Gabes, 6029 Route de Mednine Gabes, Tunisie (najla.dahmene@ fsg.rnu.tn).

§Université de Metz, Département de Mathématiques, Ile du Saulcy, 57045 Metz Cedex, France (gammella@poncelet.univ-metz.fr).

『Université de Metz, Département de Mathématiques, Ile du Saulcy, 57045 Metz Cedex, France (masmoudi@poncelet.univ-metz.fr).
} 
$E$ with an associative fiberwise product based on the Kontsevich's star product formula for $\mathbb{R}^{d}$ and with a natural connection $D=d+A_{x}\left(., \alpha_{x}\right)$, which gives a derivation of the algebra $\Gamma(E)$ of sections of $E$. Following the Fedosov's deformation quantization for symplectic manifolds $[\mathrm{F}]$, they can then modify $D$ in $\bar{D}=D+[\gamma, .]_{\star}$ so that $\bar{D}^{2}=0$, and construct a bijection $\rho$ between $C^{\infty}(M)[[\hbar]]$ and the algebra $H^{0}(E, \bar{D})$ of $\bar{D}$-horizontal sections of $E$. They finally get a star product on $M$ :

$$
f \star_{M} g=\rho^{-1}(\rho(f) \star \rho(g)) \quad \forall f, g \in C^{\infty}(M) .
$$

The purpose of the present paper is to consider that construction when $M$ is a fibred Poisson manifold and to prove that in this case the mapping $\rho$ can be chosen such that $\star_{M}$ is in fact tangential to the fibers of $M$.

A fibred Poisson manifold is a special case of foliated Poisson manifold, namely a Poisson manifold equipped with a foliation such that the Poisson tensor is tangential to the leaves. Thus, our result completes the existence proof of Masmoudi in $[\mathrm{M}]$ for tangential star products on regular Poisson manifolds. In the dual $\mathfrak{g}^{*}$ of any Lie algebra $\mathfrak{g}$, there is a dense open subset which is a foliated Poisson manifold, namely the union $\Omega$ of maximal dimensional coadjoint orbits. In some cases, our result can be used to extend the foliation of $\Omega$ to a larger open foliated Poisson submanifold of $\mathfrak{g}^{*}$. Let us recall that tangentiality was extensively studied in the case of Lie algebras ([ACG, As, B, CGR]).

The paper is organized as follows. In Section 2, we adapt the notions of formal geometry to the case of foliated and fibred Poisson manifolds. Section 3 is devoted to the construction of a preserving tangentiality section, in the formal geometrical description, on fibred Poisson manifolds. In Section 4, we describe the construction in terms of tangential objects. In Section 5, we prove that each fibred Poisson manifold admits a tangential star product. Finally, in Section 6 , we apply our result to some duals $\mathfrak{g}^{*}$ of Lie algebras.

\section{Formal geometry for foliated Poisson manifolds.}

Definition 2.1. (Foliated Poisson manifold) A smooth manifold is a foliated Poisson manifold if it is a foliated manifold and a Poisson manifold with a Poisson tensor tangential to the leaves of the foliation.

EXAMPLes 2.2. 1) If $M$ is a regular Poisson manifold, then it is foliated with the symplectic leaves thus $M$ is a foliated Poisson manifold (see [L]).

2) Let $M=\mathfrak{g}^{*}$ be the dual of the $2 n+1$-dimensional Heisenberg Lie algebra with center $\mathbb{R} Z$. The coadjoint orbits are the $2 n$-dimensional spaces:

$$
O_{\lambda}=\left\{\xi \in \mathfrak{g}^{*}:<\xi, Z>=\lambda \neq 0\right\}
$$

and the points of $F=\left\{\xi \in \mathfrak{g}^{*}:<\xi, Z>=0\right\}$. Thus, $\mathfrak{g}^{*}$ is foliated with the $O_{\lambda}$ and $F$, and is a foliated Poisson manifold.

Less elementary examples will be given in Section 6 .

A special case is the fibre bundle case. If $p: M \longrightarrow N$ is a fibre bundle with a fiber $F$, then $L_{x}=p^{-1}(p(x))$ defines a foliation $\mathcal{L}$ of $M$.

Definition 2.3. (Fibred Poisson manifold) If $p: M \longrightarrow N$ is a fibre bundle equipped with a vertical Poisson tensor $\alpha$, we say that $N$ is a fibred Poisson manifold. 
The first step of formal geometry for any manifold $M$ is to consider the 'manifold' $M^{\text {coor }}$ of jets of coordinates systems on $M$. In the following, we will always assume that $(M, \mathcal{L}, \alpha)$ is a foliated Poisson manifold (of dimension $d$, with foliation $\mathcal{L}$ and Poisson tensor $\alpha$ ) and shall only use adapted coordinates systems for $M$ i.e. local diffeomorphisms $\varphi: U \rightarrow M$ where $U$ is an open neighborhood of 0 in $\mathbb{R}^{d}$ and such that $\varphi$ defines a chart on $M$ which is adapted to the foliation $\mathcal{L}$. It means that, for any leaf $L$ of $\mathcal{L}$, each connected component $C$ of the intersection $L \cap \varphi(U)$ has the form

$$
C=\left\{\varphi(y): y^{n+1}=a_{n+1}, \ldots, y^{d}=a_{d}\right\}_{y=\left(y^{1}, \ldots, y^{d}\right) \in U},
$$

if $n$ denotes the dimension of $\mathcal{L}$.

Definition 2.4. (The manifold $M^{\text {adapt }}$ ) $M^{\text {adapt }}$ is the set of infinite jets $[\varphi]$ at 0 of local adapted charts $\varphi: U \subset \mathbb{R}^{d} \rightarrow M$.

Of course, $M^{\text {adapt }} \rightarrow M:[\varphi] \mapsto \varphi(0)$ is a fiber bundle on $M$. Its fiber is isomorphic to the group $G_{0, t}$ of infinite jets of tangential transformations $g$ preserving 0 :

$$
\begin{aligned}
g: & \mathbb{R}^{d} \rightarrow \mathbb{R}^{d}, \quad g(0)=0, \\
g\left(y^{1}, \ldots, y^{n}, a_{n+1}, \ldots, a_{d}\right) & =\left(z^{1}, \ldots, z^{n}, b_{n+1}, \ldots, b_{d}\right) \\
& =\left(z^{1}(y, a), \ldots, z^{n}(y, a), b_{n+1}(a), \ldots, b_{d}(a)\right) .
\end{aligned}
$$

The Lie algebra of $G_{0, t}$ can be written as

$$
\mathcal{W}_{0, t}=\left\{\sum_{j=1}^{n} u_{j}\left(y^{1}, \ldots, y^{d}\right) \frac{\partial}{\partial y^{j}}+\sum_{j=n+1}^{d} u_{j}\left(y^{n+1}, \ldots, y^{d}\right) \frac{\partial}{\partial y^{j}}, u_{j}(y) \in \mathbb{R}[[y]], u_{j}(0)=0 \forall j\right\} .
$$

The tangent space to $M^{\text {adapt }}$ at a point $[\varphi]$ can be identified with the Lie algebra

$$
\mathcal{W}_{t}=\left\{\sum_{j=1}^{n} u_{j}\left(y^{1}, \ldots, y^{d}\right) \frac{\partial}{\partial y^{j}}+\sum_{j=n+1}^{d} u_{j}\left(y^{n+1}, \ldots, y^{d}\right) \frac{\partial}{\partial y^{j}}, u_{j}(y) \in \mathbb{R}[[y]]\right\} .
$$

Let us introduce the usual Maurer-Cartan form $\omega_{M C}$ defined in $\Omega^{1}\left(M^{\text {adapt }}, \mathcal{W}_{t}\right)$ as follows. Let $\varphi$ be a local adapted chart and $\xi$ be in $T_{[\varphi]} M^{\text {adapt }}$. Then there exists a vector field $\tilde{\xi}$ on $M$ with infinite jet $[\tilde{\xi}]=\xi$ at $x=\varphi(0)$. Let $f_{t}$ be the flow of the vector field $\tilde{\xi}$ on $M$, thus, since $\xi$ is in $T_{[\varphi]} M^{\text {adapt }}, f_{t} \circ \varphi$ is a local adapted chart and $\omega_{M C}$ is by definition:

$$
\omega_{M C}([\varphi])(\xi)=\text { Taylor expansion at } 0 \text { of }-d \varphi(y)^{-1} \frac{d}{d t}\left(f_{t} \circ \varphi\right)_{\mid t=0} .
$$

Just as in $[\mathrm{K}]$ and $[\mathrm{CFT}]$, we need to substitute for $M^{\text {adapt }}$ a new bundle with contractible fibers. We consider the subgroup $H \subset G L(d, \mathbb{R})$ of linear transformations $h$ whose associated matrices have the form

$$
h=\left(\begin{array}{cc}
A & B \\
0 & C
\end{array}\right) .
$$


Then $H$ acts naturally (on the right) on $M^{\text {adapt }}$ and we put

$$
M_{\text {aff }}^{\text {adapt }}=M^{\text {adapt }} / H .
$$

This bundle has contractible fibers, $G_{0, t} / H$ being isomorphic to a vector space, and thus admits global sections. Note that $M^{\text {adapt }}$ (resp. $M_{\text {aff }}^{\text {adapt }}$ ) is a subbundle of the fiber bundle $M^{\text {coor }}$ (resp. $M^{\text {aff }}$ ) considered by Kontsevich in $[\mathrm{K}]$ and CattaneoFelder-Tomassini in [CFT].

Let us now choose a section $s: M \rightarrow M_{\text {aff }}^{\text {adapt }}$ of the fiber bundle $M_{\text {aff }}^{\text {adapt }}$. As in [CFT], we consider over $M_{\text {aff }}^{\text {adapt }}$ the vector bundle

$$
\tilde{E}_{0}=M^{\text {adapt }} \times_{H} \mathbb{R}\left[\left[y^{1}, \ldots, y^{d}\right]\right]
$$

and over $M$, its pull-back by $s$ :

$$
E_{0}=s \tilde{E}_{0}
$$

In fact, there is a natural injection $j: M^{\text {adapt }} \rightarrow M^{\text {coor }}$. It gives an injection $i: M_{\text {aff }}^{\text {adapt }} \rightarrow M^{\text {aff }}$. If we compose the section $s$ with $i$, we get a section $i \circ s$ of the bundle $M^{\text {aff }} \rightarrow M$ and our vector bundle $E_{0}$ coincides with the vector bundle of [CFT] which is the pull-back by $i \circ s$ of a bundle over $M^{\text {aff }}$.

On the trivial vector bundle $M^{\text {adapt }} \times \mathbb{R}\left[\left[y^{1}, \ldots, y^{d}\right]\right] \rightarrow M^{\text {adapt }}$, there is a canonical flat connection $d+\omega_{M C}$. For this connection, the horizontal sections are exactly the mappings

$$
[\varphi] \mapsto \text { Taylor expansion at } 0 \text { of } f \circ \varphi
$$

for a given $C^{\infty}$-function $f$ on $M$. By $H$-equivariance, we get simultaneously a connection, still denoted $d+\omega_{M C}$, on $\tilde{E}_{0} \rightarrow M_{\text {aff }}^{\text {adapt }}$. As in $[\mathrm{CFT}]$, we obtain by pull-back a globally well defined flat connection $D_{0}$ on $E_{0} \rightarrow M$.

3. Sections for foliated and fibred Poisson manifolds. We repeat now the preceeding construction by adding the parameter $\hbar$. We define first the bundle

$$
\tilde{E}=M^{\text {adapt }} \times_{H} \mathbb{R}\left[\left[y^{1}, \ldots, y^{d}\right]\right][[\hbar]]
$$

over $M_{\text {aff }}^{\text {adapt }}$, in the same way as $\tilde{E}_{0}$. We denote by $E$ its pull-back through the section $s: M \rightarrow M_{\text {aff }}^{\text {adapt }}$

$$
E=s \tilde{E}
$$

Let us describe a local trivialization of the bundle $E$. Let $\varphi: U \rightarrow M$ be a local adapted chart of $M$, with $U$ contractible. Using $\varphi$, we get a section

$$
s_{U}: \varphi(U) \rightarrow \varphi(U)^{\text {adapt }}
$$


as follows. If $x$ is in $\varphi(U)$, we note $U_{x}=U-\varphi^{-1}(x)$ and define a local diffeomorphism $\varphi_{x}: U_{x} \rightarrow M$ by $\varphi_{x}(y)=\varphi\left(\varphi^{-1}(x)+y\right)$ and finally the mapping $s_{U}$ by $s_{U}(x)=\left[\varphi_{x}\right]$. On $\varphi(U)^{\text {adapt }}$, we have a trivial vector bundle:

$$
\varphi(U)^{\text {adapt }} \times \mathbb{R}\left[\left[y^{1}, \ldots, y^{d}\right]\right][[\hbar]] .
$$

The pull-back of this bundle is a trivialization of $E$ over $\varphi(U)$. A section of $E$ can thus be seen as a function $x \in \varphi(U) \longmapsto f_{x} \in \mathbb{R}\left[\left[y^{1}, \ldots, y^{d}\right]\right][[\hbar]]$. Now, since all our constructions are $H$-invariant and since $\left[\varphi_{x}\right]$ is related to $s(x)$ by an element $h_{x}$ of $H$ :

$$
s(x)=s_{U}(x) \circ h_{x}=\left[\varphi_{x}\right] \circ h_{x}
$$

by $H$-equivariance, we can use this trivialization in all our computations. From now on, we thus shall present global well defined objects on $E$ with their local expression. Let $\mathcal{U}=\sum \mathcal{U}_{k}$ be the Kontsevich explicit formality on $\mathbb{R}^{d}$. Then, on each fiber of $E$, we have a Poisson structure:

$$
\alpha_{x}=\left(\varphi_{x}^{-1}\right)_{*}(\alpha) .
$$

An associative fiberwise product for $E$ is given by the Kontsevich's star product formula computed 'in the variables $y$ ':

$$
f_{x} \star g_{x}=P_{x}^{M}(\alpha)\left(f_{x} \otimes g_{x}\right)
$$

where

$$
P_{x}^{M}(\alpha)=\sum_{k \geq 0} \frac{\hbar^{k}}{k !} \mathcal{U}_{k}\left(\alpha_{x} \ldots \ldots \alpha_{x}\right) .
$$

By construction, $\star$ is tangential in the sense that each $\mathcal{U}_{k}\left(\alpha_{x} \ldots \ldots \alpha_{x}\right)$ is in our coordinates a bidifferential operator with derivations only with respect to the tangential variables $y^{1}, \ldots, y^{n}$. Then, we extend as in [CFT] the connection $D_{0}$ to a connection $D$ on $E$ by putting:

$$
(D f)_{x}=d_{x} f+A_{x}^{M} f_{x}
$$

where if $\xi$ is in $T_{x} M, A_{x}^{M}(\xi)$ means

$$
A_{x}^{M}(\xi)=A\left(s^{*}\left(\omega_{M C}\right)_{x}(\xi), \alpha_{x}\right)=\sum_{k \geq 0} \frac{\hbar^{k}}{k !} \mathcal{U}_{k+1}\left(s^{*}\left(\omega_{M C}\right)_{x}(\xi) \cdot \alpha_{x} \ldots \ldots \alpha_{x}\right) .
$$

Since we want to build tangential operators, $A_{x}^{M}(\xi)$ has to be tangential if $\xi$ itself is tangent to the leaf containing $x$. This property holds surely if $s^{*}\left(\omega_{M C}\right)_{x}(\xi)$ is a vector in $\sum_{j=1}^{n} \mathbb{R}\left[\left[y^{1}, \ldots, y^{d}\right]\right][[\hbar]] \frac{\partial}{\partial y^{j}}$. Locally, we can choose $s$ such that this property holds: the section $s_{U}$ of $\varphi(U)$ satisfies this relation. But this does not hold for any $s$. For instance :

If $M=\mathbb{R}^{2}$ with the foliation $\mathcal{L}$ defined by :

$$
L_{(x, y)}=\{(x+z, y), \quad z \in \mathbb{R}\}
$$

and $\xi=\left.\partial_{x}\right|_{(x, y)}$. Let $s(x, y)=\left[\varphi_{(x, y)}\right]$ be a section of $M^{\text {adapt }}$. We put

$$
\varphi_{(x, y)}(u, v)=\left(f_{(x, y)}(u, v), g_{(x, y)}(v)\right) .
$$


We choose $\gamma(t)=(x+t, y)$ then $\left.\frac{d}{d t}\right|_{t=0} \gamma(t)=\xi$ and

$$
\begin{aligned}
\left(\varphi_{(x, y)}\right)^{-1}\left(\varphi_{\gamma(t)}\right)(u, v) & =\left(u^{\prime}, v^{\prime}\right) \\
& =\left(\left(p r_{1} \circ f_{(x, y)}\right)^{-1}\left(f_{(x+t, y)}(u, v), g_{(x+t, y)}^{-1}\left(g_{(x+t, y)}(v)\right)\right)\right) \\
& =(F(t, u, v), G(t, v)) .
\end{aligned}
$$

We get the value of the Maurer-Cartan form in $s(x, y)$ on $s_{*}(\xi)$ :

$\omega_{M C}(s(x, y))\left(s_{*}(\xi)\right)=$

[Taylor exp. in $(u, v)$ of $\left.\frac{\partial F}{\partial t}(0, u, v)\right] \frac{\partial}{\partial u}+\left[\right.$ Taylor exp. in $(u, v)$ of $\left.\frac{\partial G}{\partial t}(0, v)\right] \frac{\partial}{\partial v}$.

Thus $s^{*}\left(w_{M C}\right)_{(x, y)}(\xi)$ is in $\mathbb{R}[[u, v]][[\hbar]] \frac{\partial}{\partial u}$ only if $\frac{\partial G}{\partial t}(0, v) \equiv 0$. This relation holds if our charts preserve the fibre bundle structure of $\mathbb{R}^{2} \rightarrow \mathbb{R} \quad(\quad(x, y) \mapsto y \quad)$ :

$$
\varphi_{(x, y)}(u, v)=\left(f_{(x, y)}(u, v), g_{y}(v)\right) .
$$

We are very grateful to our referee for pointing us this fact: in our first version, we were not aware of it.

Definition 3.1. (Preserving tangentiality sections) Let $(M, \mathcal{L})$ be a foliated manifold and $s: M \rightarrow M^{\text {adapt }}$ (resp. $s: M \rightarrow M_{\text {aff }}^{\text {adapt }}$ ) a section of the bundle $M \rightarrow M^{\text {adapt }}$ (resp. $M \rightarrow M_{\text {aff }}^{\text {adapt }}$ ). We say that s preserves tangentiality if $s^{*}\left(\omega_{M C}\right)_{x}(\xi)$ is a vector in $\sum_{j=1}^{n} \mathbb{R}\left[\left[y^{1}, \ldots, y^{d}\right]\right][[\hbar]] \frac{\partial}{\partial y^{j}}$ for any $x$ and any $\xi$ itself tangent to the leaf containing $x$.

Let us consider now the case of fibre bundle manifolds.

Let $p: M \rightarrow N$ be a fibre bundle with fiber $F$ and $s^{N}: N \rightarrow N^{\text {adapt }}, s^{F}: F \rightarrow$ $F^{\text {adapt }}$ sections of the bundles $N^{\text {adapt }} \rightarrow N, F^{\text {adapt }} \rightarrow F$.

For any $p(x)$ in $N$, we choose a chart $\left(V, \varphi_{p(x)}^{N}\right)$ such that $s^{N}(p(x))=\left[\varphi_{p(x)}^{N}\right]$ and there is a local trivialization :

$$
\Psi_{\varphi_{p(x)}^{N}(V)}: p^{-1}\left(\varphi_{p(x)}^{N}(V)\right) \rightarrow \varphi_{p(x)}^{N}(V) \times F, \quad u \mapsto\left(p(y), \Theta_{\varphi_{p(x)}^{N}(V)}(y)\right) .
$$

From now on, we denote by $\Theta$ the mapping $\Theta_{\varphi_{p(x)}^{N}(V)}$, if there is no possible confusion.

Similarly, for any $z$ in $F$, we choose a chart $\left(W, \varphi_{z}^{F}\right)$ such that $s^{F}(z)=\left[\varphi_{z}^{F}\right]$. Let us define the local chart of $M$ centred in $x$ by :

$$
\begin{aligned}
\varphi_{x}^{M}: U=V \times W & \longrightarrow M \\
(v, w) & \longmapsto\left(\Psi_{\varphi_{p(x)}^{N}}(V)\right)^{-1}\left(\varphi_{p(x)}^{N}(v), \varphi_{\Theta(x)}^{F}(w)\right) .
\end{aligned}
$$

Now for any $g_{p(x)}^{V}$ and $g_{z}^{W}$ in $G L(d-n)$ (resp. in $\left.G L(n)\right)$, we have

$$
s^{N}(p(x))=\left[\varphi_{p(x)}^{N} \circ g_{p(x)}^{V}\right]
$$

and

$$
s^{F}(z)=\left[\varphi_{z}^{F} \circ g_{z}^{W}\right]
$$


Then we consider $\varphi_{x}^{M} \circ g_{x}^{U}$ where $g_{x}^{U}=\left(\begin{array}{cc}g_{p(x)}^{V} & 0 \\ 0 & g_{z}^{W}\end{array}\right)$.

Proposition 3.2. (Good sections for fibre bundles) The section $s^{M}$ of the bundle $M^{\text {adapt }}$ (resp. $M_{\text {aff }}^{\text {adapt }}$ ) defined by $s^{M}(x)=\left[\varphi_{x}^{M}\right]\left(\right.$ resp. $\left.s^{M}(x)=\left[\varphi_{x}^{M} \circ g_{x}^{U}\right]\right)$ satisfies:

$$
s_{*}^{M}(\xi)=\left(0, s_{*}^{F} \circ \Theta_{*}(\xi)\right)
$$

for any vertical vector $\xi$ in $T_{x}(M)$. In other words, $s^{M}$ preserves tangentiality.

Proof. Let $\xi$ be a vertical vector in $T_{x}(M)$. We consider a curve $t \mapsto \gamma(t)$ such that :

$$
\theta(0)=x, \quad p(\gamma(t))=p(x),\left.\quad \frac{d}{d}\right|_{t=0} \gamma(t)=\xi
$$

We have

$$
\varphi_{\gamma(t)}^{M}(v, w)=\left(\Psi_{\varphi_{p(x)}}^{N}(V)\right)^{-1}\left(\varphi_{p(x)}^{N}(v), \varphi_{\Theta(\gamma(t))}^{F}(w)\right)
$$

Then

$$
\left(\varphi_{x}^{M}\right)^{-1}\left(\varphi_{\gamma(t)}^{M}(v, w)\right)=\left(v,\left(\varphi_{\Theta(x)}^{F}\right)^{-1}\left(\varphi_{\Theta(\gamma(t))}^{F}(w)\right)\right)
$$

By derivation, we get

$$
s_{*}^{M}(\xi)=s_{*}^{M}\left(\left.\frac{d}{d t}\right|_{t=0} \gamma(t)\right)=\left(0, s_{*}^{F} \circ \Theta_{*}(\xi)\right) .
$$

If we have only sections for the bundles $N$ and $F$, then for any $g_{p(x)}^{V}$ and $g_{\gamma(t)}^{W}$, we get

$$
\left(\varphi_{x}^{M} \circ g_{x}^{U}\right)^{-1}\left(\varphi_{\gamma(t)}^{M} \circ g_{\gamma(t)}^{U}(v, w)\right)=\left(v,\left(g_{\Theta(x)}^{W}\right)^{-1} \circ\left(\varphi_{\Theta(x)}^{F}\right)^{-1}\left(\varphi_{\Theta(\gamma(t))}^{F} g_{\gamma(t)}^{W}(w)\right)\right) .
$$

4. Fiberwise deformation on $E_{0}$. From now on, we restrict ourselves to the case of a foliated Poisson manifold $(M, \mathcal{L}, \alpha)$ for which the bundle $M_{\text {aff }}^{\text {adapt }}$ has a section which preserves tangentiality. Therefore, we see that all the differential operators $\mathcal{U}_{k+1}\left(s^{*}\left(\omega_{M C}\right)_{x}(\xi) \cdot \alpha_{x} \ldots \alpha_{x}\right)$ are tangential, and so is $A_{x}^{M}(\xi)$.

Now, as in $[\mathrm{F}]$ and $[\mathrm{CFT}]$, we extend the above fiberwise product $\star$ to a product, still denoted $\star$, on the space $\Omega^{\bullet}(M, E)=\Omega^{\bullet}(M) \otimes_{C^{\infty}(M)} \Gamma(E)$ of forms on $M$ with values in the fiber by putting on $\varphi(U)$ :

$$
\begin{aligned}
\omega \star \omega^{\prime} & =\left(a_{i_{1}, \ldots, i_{k}}(y) d x^{i_{1}} \wedge \ldots \wedge d x^{i_{k}}\right) \star\left(b_{j_{1}, \ldots, j_{l}}(y) d x^{j_{1}} \wedge \ldots \wedge d x^{j_{l}}\right) \\
& =\left(a_{i_{1}, \ldots, i_{k}} \star b_{j_{1}, \ldots, j_{l}}\right)(y) d x^{i_{1}} \wedge \ldots \wedge d x^{i_{k}} \wedge d x^{j_{1}} \wedge \ldots \wedge d x^{j_{l}} .
\end{aligned}
$$

We extend also the connection $D$ (and $\left.D_{0}\right)$ to $\Omega^{\bullet}(M, E)$ by

$$
D(a b)=\left(d_{x} a\right) b+(-1)^{p} a D b \quad \forall a \in \Omega^{p}(M), \forall b \in \Omega^{\bullet}(M, E),
$$


or, equivalently,

$$
\begin{aligned}
D \omega\left(X_{0}, \ldots, X_{p}\right)= & \sum_{i=0}^{p} D_{X_{i}}\left(\omega\left(X_{0}, \ldots, X_{p}\right)\right)+ \\
& +\sum_{i<j}(-1)^{i+j} \omega\left(\left[X_{i}, X_{j}\right], X_{0}, \ldots, \hat{X}_{i}, \ldots, \hat{X}_{j}, \ldots, X_{p}\right),
\end{aligned}
$$

if $\omega \in \Omega^{\bullet}(M, E)$ and the $X_{i}$ are vector fields on $M$.

One can then prove

TheOrem 4.1.[CFT] (Properties of $\star$ and $D$ ) Let $F^{M}$ be the $E$ valued-two-form defined by

$$
\begin{aligned}
F_{x}^{M}(\xi, \eta) & =F\left(s^{*}\left(\omega_{M C}\right)_{x}(\xi), s^{*}\left(\omega_{M C}\right)_{x}(\eta)\right) \\
& =\sum_{k \geq 0} \hbar^{k} \frac{1}{k !} \mathcal{U}_{k+2}\left(s^{*}\left(\omega_{M C}\right)_{x}(\xi) \cdot s^{*}\left(\omega_{M C}\right)_{x}(\eta) \cdot \alpha_{x} \ldots \alpha_{x}\right) .
\end{aligned}
$$

Then, for any $f, g$ in $\Gamma(E)$, the following relations hold

$$
\begin{aligned}
& \text { i) } D(f \star g)=(D f) \star g+f \star(D g) \\
& \text { ii) } D^{2}(f)=F^{M} \star f-f \star F^{M} \\
& \text { iii) } D F^{M}=0 \quad \text { (Bianchi identity). }
\end{aligned}
$$

Let us now introduce the notion of tangential Fedosov connections.

Definition 4.2. A Fedosov connection $D$ on $E$, with Weyl curvature $F$ in $\Omega^{2}(M, E)$, is a connection on $E$ such that for any $\omega, \omega^{\prime}$ in $\Omega^{\bullet}(M, E)$,

$$
D\left(\omega \star \omega^{\prime}\right)=(D \omega) \star \omega^{\prime}+(-1)^{\operatorname{deg}(\omega)} \omega \star\left(D \omega^{\prime}\right), D^{2} \omega=F \star \omega-\omega \star F, \text { and } D F=0 .
$$

A Fedosov connection $D=d+\Theta$ is tangential (to the foliation $\mathcal{L}$ of $M$ ) if, for any vector $\xi$ tangential to $\mathcal{L}, \Theta(\xi)$ is $\overline{\text { a formal }}$ tangential differential operator on $\mathbb{R}\left[\left[y^{1}, \ldots, y^{d}\right]\right]$.

It follows from Theorem 4.1 and Definition 4.2 that our natural connection $D$ on $\Omega^{\bullet}(M, E)$ is a tangential Fedosov connection with Weyl curvature $F^{M}$.

5. Flat Fedosov connections and star products. Following the idea of Fedosov, we now modify $D$ in order to get a flat tangential Fedosov connection. First, we have

Proposition 5.1. (Cohomology of $\left(E_{0}, D_{0}\right)$ ) The cohomology groups of $D_{0}$ : $\Omega^{\bullet}\left(M, E_{0}\right) \rightarrow \Omega^{\bullet+1}\left(M, E_{0}\right)$ are

$$
H^{p}\left(E_{0}, D_{0}\right)=\left\{\begin{array}{ccc}
C^{\infty}(M) & \text { if } & p=0 \\
\{0\} & \text { if } & p>0 .
\end{array}\right.
$$

See a detailled proof in $[\mathrm{F}]$ and $[\mathrm{CFT}]$ (Appendix B).

Proposition 5.2. There exists a flat tangential Fedosov connection on E. 
Proof. We look for a flat connection $\bar{D}$ of the form

$$
\bar{D}=D+(\gamma \star .-. \star \gamma)=D+[\gamma,]_{\star}
$$

where

$$
\gamma=\hbar \gamma_{1}+\ldots+\hbar^{k} \gamma_{k} \ldots \in \Omega^{1}(M, E) .
$$

The flatness of $\bar{D}$ is equivalent to the equation

$$
F^{M}+D \gamma+\gamma \star \gamma=0 .
$$

Since $F^{M}=\hbar F_{1}^{M}+\hbar^{2} F_{2}^{M}+\ldots$ and $H^{2}\left(E_{0}, D_{0}\right)=\{0\}$, we can, as usual, solve the above equation recursively in $\gamma_{k}$. With that solution $\gamma, \bar{D}$ is a flat Fedosov connection and since $D$ is tangential, $\bar{D}$ is tangential too. With a flat Fedosov connection $\bar{D}$, it is possible to define a star product on $M$. We recall here the construction. Let us first consider the canonical application $\pi$ from $H^{0}(E, \bar{D})$ to $C^{\infty}(M)$ assigning to each flat section $f$ on $E$ the $C^{\infty}$-function $x \mapsto f_{x}(0)$. We have then to find a quantization mapping i.e. a section $\rho$ of $\pi$ of the form:

$$
\rho=i d+\hbar \rho_{1}+\ldots: H^{0}\left(E_{0}, D_{0}\right) \simeq C^{\infty}(M) \rightarrow H^{0}(E, \bar{D}) .
$$

By standard arguments (see $[\mathrm{F}]$ and $[\mathrm{CFT}]$ ), such a mapping $\rho$ always exists. In fact, if $T$ is a differentiel operator on $E_{0}$, we put $D_{0}(T)=D_{0} \circ T-T \circ D_{0}$. Since $D_{0}$ is flat, we get a new cohomology. If we restrict ourselves to the space $B_{k}$ of differential operators of order $\geq k$, vanishing on constants, then

$$
H^{p}\left(B_{k}, D_{0}\right)=0 \quad \forall k \geq 0, \forall p>0 .
$$

The vanishing of $H^{1}\left(B_{k}, D_{0}\right)$ for all $k$ enables us to construct recursively a solution $\rho$ of the equation

$$
D \circ \rho-\rho \circ D_{0}=0 .
$$

The star product is then given by:

$$
f \star_{M} g=\pi(\rho(f) \star \rho(g)) \quad \forall f, g \in C^{\infty}(M) .
$$

In [CFT], there is also a more explicit construction of the mapping $\rho$. In the following, we shall use this second construction to get a tangential star product on $M$.

Definition 5.3. A star product $\star$ on the foliated Poisson manifold $(M, \mathcal{L}, \alpha)$ is tangential, if for the domain $V$ of any local adapted chart and for all function $f$ constant on the plaques,

$$
f \star g_{\mid V}=f g_{\mid V} \quad \forall g \in C^{\infty}(M) .
$$

We want now to prove the main result of the paper.

THEOREM 5.4. (Tangentiality of $\star_{M}$ ) If $M_{\text {aff }}^{\text {adapt }}$ has a preserving tangentiality section $s$, the section $\rho$ can be chosen in such a manner that $\star_{M}$ is a tangential star product on $M$. 
Proof. As in $[\mathrm{CFT}]$, let us put

$$
R_{x}\left(f_{x}, \alpha_{x}\right)=\sum_{k \geq 0} \hbar^{k} \frac{1}{k !} \mathcal{U}_{k+1}\left(f_{x} \cdot \alpha_{x} \ldots \ldots \alpha_{x}\right) \in \mathbb{R}\left[\left[y^{1}, \ldots, y^{d}\right]\right][[\hbar]] .
$$

For any Hamiltonian vector field $\eta$ on $M$ with Taylor expansion $\bar{\eta}$, there exists a unique $\beta(\eta)$ in $\Omega^{0}(M, E)=\Gamma(E)$ such that

$$
\begin{aligned}
& \bar{D} \beta(\eta) \quad=-F\left(\bar{\eta}, s^{*}\left(\omega_{M C}(.)\right), \alpha_{x}\right)+A\left(\bar{\eta}, \alpha_{x}\right) \gamma \\
& \beta(\eta)(y=0) \quad=\quad 0
\end{aligned}
$$

By construction, $\eta \rightarrow \beta(\eta)$ is linear and the mapping $\rho$ defined by

$$
\rho(f)(x)=R_{x}\left(f_{x}, \alpha_{x}\right)+\hbar \beta([\alpha, f])(x)
$$

is a quantization mapping $([\mathrm{CFT}])$.

It remains thus to show that the star product induced by this map $\rho$ on $M$ is tangential. Let us choose any domain $V$ of local adapted chart and $f$ a local function constant on the plaques of $V$. We need to prove that $\rho(f)=f$. First, we see that

$$
\mathcal{U}_{k+1}\left(f_{x} \cdot \alpha_{x} \ldots \alpha_{x}\right)=0 \quad \forall k \geq 1
$$

Indeed, this function is (see $[\mathrm{K}])$ :

$$
\mathcal{U}_{k+1}\left(f_{x} \cdot \alpha_{x} \ldots \alpha_{x}\right)=\sum_{\Gamma \in G_{k+1,0}} W_{\Gamma} B_{\Gamma}\left(f_{x} \cdot \alpha_{x} \ldots \alpha_{x}\right),
$$

where $G_{k+1,0}$ is the set of admissible graphs $\Gamma$ with $k+1$ vertices $p_{0}, p_{1}, \ldots, p_{k}$ in the Poincaré half plane

$$
\mathcal{H}=\{z \in \mathbf{C}: \Im(z)>0\}
$$

and $2 k$ edges: two edges starting from each $p_{i}, i \geq 1$ and ending to any another vertex (there is no double edge). We recall that $B_{\Gamma}\left(f_{x} \cdot \alpha_{x} \ldots \alpha_{x}\right)$ is a function defined, starting from $\Gamma$, by partial derivatives of $f_{x}$ and of the components $\alpha_{x}^{i j}$. Since $f$ is constant on the leaves and $\alpha$ tangential, the only graphs $\Gamma$ for which $B_{\Gamma}\left(f_{x} . \alpha_{x} \ldots \alpha_{x}\right)$ does not vanish are graphs where no edge ends at $p_{0}$. Moreover, by definition, the wedge $W_{\Gamma}$ is the integral on a configuration space $C_{k+1,0}^{+}$of a $2 k$-form $\omega_{\Gamma}$. The latter is a product of 1 -forms $d \phi$, each of these 1 -forms corresponding to exactly one edge of $\Gamma$. Generally speaking, for each non connected graph $\Gamma$, the wedge $W_{\Gamma}$ is zero. For our special graphs $\Gamma$, this can be easily seen. Let us parametrize $C_{k+1,0}^{+}$by putting

$$
p_{1}=i, p_{0}=z_{0}, p_{2}=z_{1}, \ldots, p_{k}=z_{k-1} .
$$

Then, we can identify $C_{k+1,0}^{+}$with an open dense subset of $C_{k, 0}^{+} \times \mathcal{H}^{+}$by means of

$$
\left(i, z_{0}, z_{1}, \ldots, z_{k-1}\right) \mapsto\left(\left(i, z_{1}, \ldots, z_{k-1}\right), z_{0}\right) .
$$

For each of our graphs $\Gamma$, the $2 k$-form $\omega_{\Gamma}$ does not depend on $z_{0}$ and thus belongs to $\bigwedge^{2 k}\left(C_{k, 0}^{+}\right)$. Since $C_{k, 0}^{+}$is of dimension $2 k-2$, we have $\bigwedge^{2 k}\left(C_{k, 0}^{+}\right)=\{0\}$ and $\omega_{\Gamma}=0$. Finally, since $f$ is constant on the leaves of $\mathcal{L}$ by assumption and $\alpha$ tangential, $[\alpha, f]=0$. Moreover, by linearity of $\beta, \beta([\alpha, f])=0$. Thus, as announced,

$$
\rho(f)(x)=\mathcal{U}_{1}\left(f_{x}\right)=f_{x} \quad \forall x \in V .
$$


Now, by tangentiality of $\star$ in the variables $y$, we have fiber by fiber the equality

$$
f \star \rho(g)(x)=f_{x} \rho(g)(x) \quad \forall g \in C^{\infty}(M), \forall x \in V .
$$

Computing $\rho(f g)$, we get

$$
\rho(f g)(x)=R_{x}\left(f_{x} g_{x}, \alpha_{x}\right)+\hbar \beta([\alpha, f g])(x)=f_{x} R_{x}\left(g_{x}, \alpha_{x}\right)+\hbar \beta(f[\alpha, g])(x) \quad \forall x \in V .
$$

Furthermore, for any Hamiltonian vector field $\eta^{\prime}=f \eta, f \beta(\eta)$ and $\beta\left(\eta^{\prime}\right)$ are two solutions of the equation:

$$
\bar{D} \beta\left(\eta^{\prime}\right)=-F\left(\overline{\eta^{\prime}}, s^{*}\left(\omega_{M C}(.)\right), \alpha_{x}\right)+A\left(\overline{\eta^{\prime}}, \alpha_{x}\right)
$$

satisfying the normalization condition $f \beta(\eta)=\beta\left(\eta^{\prime}\right)=0_{\mid y=0}$. The reason for this is that the right side of the equation is linear in $f$, by definition of the local Kontsevich formality. By uniqueness of the solution, we obtain $\beta(f \eta)=f \beta(\eta)$ and for any $g$

$$
\rho(f g)=f \rho(g) .
$$

Thus,

$$
f \star_{M} g_{\mid V}=\pi(\rho(f) \star \rho(g))_{\mid V}=\pi(f \rho(g))_{\mid V}=\pi(\rho(f g))_{\mid V}=f g_{\mid V} .
$$

This ends the proof.

6. A direct application to the dual of Lie algebra. The dual $\mathfrak{g}^{*}$ of a Lie algebra is a Poisson manifold whose symplectic (generalized) foliation is given by the coadjoint orbits. The dense open subset $\Omega$ of maximal dimensional orbits is a regular Poisson manifold. Thus, on $\Omega$, there exists tangential (to the orbits) star products.

However, there is generally no star product on the whole dual $\mathfrak{g}^{*}$ whose restriction to $\Omega$ is tangential. More precisely, M. Cahen, S. Gutt and J. Rawnsley proved in [CGR] that the existence of such a star product forces each invariant quadratic function on $\mathfrak{g}^{*}$ to satisfy a very strong algebraic condition. Especially, no semi simple Lie algebra satisfies this condition.

We want now to give concrete and nontrivial examples of nilpotent Lie algebras for which our construction can be used.

1. Let us first consider the Lie algebra $\mathfrak{g}_{5,4}$ with basis $\left(X_{1}, X_{2}, X_{3}, X_{4}, X_{5}\right)$. The nonvanishing brackets are:

$$
\left[X_{5}, X_{4}\right]=X_{3}, \quad\left[X_{5}, X_{3}\right]=X_{2}, \quad\left[X_{4}, X_{3}\right]=X_{1} .
$$

The orbit of a point $\xi=\sum \xi_{i} X_{i}^{*}$ in the dual $\mathfrak{g}_{5,4}^{*}$ is either of dimension 2 (in fact, $\Omega$ is $\Omega=\left\{\xi, \xi_{1}^{2}+\xi_{2}^{2}+\xi_{3}^{2} \neq 0\right\}$ ) or of dimension 0 (if $\xi_{1}^{2}+\xi_{2}^{2}+\xi_{3}^{2}=0$ ). The generic orbits are those of the points $\xi$ with $\xi_{1} \neq 0$, they can be parametrized with obvious notations by:

$$
O_{\xi}=\left\{\left(\xi_{1}, \xi_{2}, q, \xi_{1} p, \xi_{2} p+\lambda-\frac{q^{2}}{2 \xi_{1}}\right): p, q \in \mathbb{R}\right\} .
$$

The quadratic invariant function

$$
\mu: \xi \mapsto \lambda \xi_{1}=\xi_{5} \xi_{1}-\xi_{2} \xi_{4}+\frac{\xi_{3}^{2}}{2}
$$


corresponds to a nondegenerate quadratic form. Now, $\mathfrak{g}_{5,4}$ being a 3 -step nilpotent Lie algebra, the condition of [CGR] is not satisfied by $\mu$ and there is no star product on the whole dual $\mathfrak{g}_{5,4}^{*}$ whose restriction to $\Omega$ is tangential. In fact, since the tangent space to a generic orbit $O_{\xi}$ is

$$
T_{\xi} O_{\xi}=\left\{a \partial_{3}+b \partial_{4}+c \partial_{5}: \xi_{3} a-\xi_{2} b+\xi_{1} c=0\right\},
$$

one can directly see that the generic symplectic foliation cannot be completed to any singular coadjoint orbit.

Our result can be applied to the dense subset :

$$
\Omega_{0}=\left\{\xi, \xi_{1}^{2}+\xi_{2}^{2} \neq 0\right\}
$$

of $\Omega$. Indeed $\Omega_{0}$ is a fibred Poisson manifold : let us write any $\xi$ in $\Omega_{0}$ as

$$
\xi=\left(r \cos \theta, r \sin \theta, q,(r \cos \theta) p+\left(\frac{q^{2}}{2 r}+\lambda\right) \sin \theta,(r \sin \theta) p-\left(\frac{q^{2}}{2 r}+\lambda\right) \cos \theta\right) .
$$

Then $\Omega_{0}$ is fibred on $\mathbb{C}^{2} \backslash\{0\} \times \mathbb{R}$ by :

$$
p: \xi \mapsto\left(r \xi^{i \theta}, \lambda\right),
$$

the fibers being the coadjoint orbits.

2. Let us now consider the semi-direct product $\mathfrak{g}_{6,14}=\mathbb{R} \times \mathfrak{g}_{5,4}$ of $\mathfrak{g}_{5,4}$ by $\mathbb{R}$ with basis $\left(X_{1}, \ldots, X_{6}\right)$ and commutation relations

$$
\left[X_{6}, X_{5}\right]=X_{1}, \quad\left[X_{5}, X_{4}\right]=X_{3}, \quad\left[X_{5}, X_{3}\right]=X_{2}, \quad\left[X_{4}, X_{3}\right]=X_{1} .
$$

Then, the set of the generic orbits $O_{\xi}$ coincides with $\Omega=\left\{\xi=\sum \xi_{i} X_{i}^{*}: \xi_{1} \neq 0\right\}$ and for $\xi$ in $\Omega$, we have with obvious notations:

$$
O_{\xi}=\left\{\left(\xi_{1}, \xi_{2}, q_{1}, \xi_{1} p_{1}, \xi_{2} p_{1}-\frac{q_{1}^{2}}{2 \xi_{1}}+q_{2}, \xi_{1} p_{2}\right): p_{1}, q_{1}, p_{2}, q_{2} \in \mathbb{R}\right\} .
$$

In this case, it is possible to extend the symplectic foliation $\mathcal{L}$ into a foliation $\mathcal{L}^{\prime}$ for all $\mathfrak{g}_{1}^{*}$ by setting

$$
\mathcal{L}^{\prime}=\left(\underset{\xi_{1} \neq 0}{\cup} O_{\xi}\right) \bigcup\left(\cup_{\xi_{2}}\left\{\left(0, \xi_{2}, x, y, z, t\right): x, y, z, t \in \mathbb{R}\right\}\right) .
$$

It means that $\mathfrak{g}_{6,14}^{*}$ is a foliated Poisson manifold. The usual coordinates of $\mathbb{R}^{4}$ define on $\mathfrak{g}_{6,14}^{*}$ a global adapted chart $(U, \varphi)$ thus $s_{U}$ is a preserving tangentiality section of $\left(\mathfrak{g}_{6,14}^{*}\right)^{\text {adapt }}$.

Let us remark that $\mathfrak{g}_{6,14}^{*}$ is also a fibred Poisson manifold and by Theorem 5.4, it admits a tangential star product. Note that in this example, the Gutt star product [G] coincides with the Kontsevich-Duflo star product [A], and is already tangential.

3. Let us consider finally the 7-dimensional Lie algebra, say $\mathfrak{g}_{7}$, defined by the following brackets

$$
\left[X_{7}, X_{6}\right]=X_{1}, \quad\left[X_{5}, X_{4}\right]=X_{3}, \quad\left[X_{5}, X_{3}\right]=X_{2}, \quad\left[X_{4}, X_{3}\right]=X_{1} .
$$


Let us put :

$$
\begin{aligned}
& \xi_{1}=r \cos \theta, \xi_{2}=r \sin \theta, \xi_{3}=q_{1}, \\
& \xi_{4}=r \cos \theta p_{1}+\left(\frac{q_{1}^{2}}{2 r}+\frac{\mu}{r}\right) \sin \theta \\
& \xi_{5}=r \sin \theta p_{1}-\left(\frac{q_{1}^{2}}{2 r}+\frac{\mu}{r}\right) \cos \theta \\
& \xi_{6}=q_{2}, \quad \xi_{7}=(r \cos \theta) p_{2} .
\end{aligned}
$$

The quadratic form $F$ associated to the invariant quadratic function

$$
\xi \mapsto \mu=\xi_{1} \xi_{5}-\xi_{2} \xi_{4}-\frac{1}{2} \xi_{3}^{2}
$$

is such that $\operatorname{Ker}(F)^{\perp}=<X_{1}, X_{2}, X_{3}, X_{4}, X_{5}>$ is not 2-step nilpotent. Therefore, the condition of $[\mathrm{CGR}]$ is not satisfied and there is no tangential star product, differential and well-defined on the whole dual $\mathfrak{g}_{7}^{*}$. However, the mapping

$$
p: \xi \mapsto(r, \theta, \mu)
$$

defines a fibration of

$$
\Omega=\left\{\xi ; \xi_{1}^{2}+\xi_{2}^{2} \neq 0\right\}
$$

on $] 0 ;+\infty\left[\times S^{1} \times \mathbb{R}\right.$; the fiber being, if $\cos \theta \neq 0$, the coadjoint orbit $O_{\xi}$ and, if $\cos \theta=0$, the 4 -dimensional manifold :

$$
\left\{\left(0, r \sin \theta, x, \frac{-1}{\sin \theta}\left(\frac{\mu}{r}+\frac{x^{2}}{2 r}\right), y, z, t\right)\right\} .
$$

It is thus a fibration with fiber $F \simeq \mathbb{R}^{4}$ since

$$
p^{-1}(r, \theta, \mu)=\left\{r \cos \theta, r \sin \theta, x, \frac{-1}{\sin \theta}\left(\frac{\mu}{r}+\frac{x^{2}}{2 r}-y \cos \theta\right), y, z, t\right\}
$$

when $\sin \theta \neq 0$. Our Theorem 5.4 can be applied for $\Omega$; let us remark that the fiber in $\cos \theta=0$ is a set of 2 -dimensional orbits.

REMARKS 6.1. There is examples where star products on the whole dual $\mathfrak{g}^{*}$ exist, while it is impossible to enlarge the foliation $\mathcal{L}$ of $\Omega$. An example is given by the 'filiform' (nilpotent) Lie algebra $\mathfrak{g}_{4,1}$ whose nonvanishing brackets are

$$
\left[X_{4}, X_{3}\right]=X_{2}, \quad\left[X_{4}, X_{2}\right]=X_{1} .
$$

In this case, we cannot extend the foliation $\mathcal{L}$ to points $\xi$ in $\mathfrak{g}_{4,1}^{*}$ such that $\xi_{1}=\xi_{2}=0$. Nevertheless, since this Lie algebra is special, there exists a differential star product, globally defined on all the dual $\mathfrak{g}^{*}$ and tangential to the maximal dimensional orbits (see $[A C G, B])$.

This work on foliated and fibred Poisson manifolds raises the general problem, for a Lie algebra $\mathfrak{g}$, to extend the canonical foliation $\mathcal{L}$ outside of the set $\Omega$ of maximal dimensional orbits in $\mathfrak{g}^{*}$. We do not have yet any characterization of Lie algebras for which such an extension is possible.

Acknowledgements. The second author N. Dahmene thanks the C.M.C.U. for financial support. 


\section{REFERENCES}

[A] D. ARnal, Le produit star de Kontsevich sur le dual d'une algèbre de Lie nilpotente, C.R. Acad. Sci. Paris Ser. I Math., 327 (1998), pp. 823-826.

[ACG] D. Arnal, M. Cahen and S. Gutt, Deformations on coadjoint orbits, J. Geom. Phys., 3:3 (1986), pp. 327-351.

[As] S.L. Asin, Ph.D. Thesis, Warwick University (1998).

[B] N. Ben Amar, Tangential deformations on the dual of nilpotent special Lie algebras, Pacific J. Math., 170:2 (1995), pp. 297-318.

[CGR] M. Cahen, S. Gutt and J. Rawnsley, On tangential star products for the coadjoint Poisson structure, Comm. Math. Phys., 180 (1996), pp. 99-108.

[CFT] A.S. Cattaneo, G. Felder and L. Tomassini, From local to global deformation quantization of Poisson manifolds, Preprint QA/0012228 (2000).

[G] S. GUTt, An explicit *-product on the cotangent bundle of a Lie group, Lett. Math. Phys., 7 (1983), pp. 249-258.

[F] B.V. Fedosov, A simple geometrical construction of deformation quantization, J. Diff. Geom., 40 (1994), pp. 213-238; Deformation quantization and index theory, Akademie Verlag, Berlin 1996.

[K] M. Kontsevich, Deformation quantization of Poisson manifolds, Preprint QA/9709040 (1997).

[L] A. Lichnerowicz, Variétés de Poisson et feuilletages, Ann. Fac. Toulouse (1982), pp. 195262.

[M] M. Masmoudi, Tangential formal deformations of the Poisson bracket and tangential star products on a regular Poisson manifold, J. Geom. Phys., 9 (1992), pp. 155-171. 